\title{
Gun control saves lives
}

In the January edition of $S A M J$, Prinsloo et al.$^{[1]}$ reported that data from the police and a recent study of injury mortality based on postmortem investigations showed a significant decrease in homicide in South Africa (SA). In 2000, SA had one of the world's highest homicide rates, estimated at 67 per 100000 population, ${ }^{[2]}$ but this had almost halved by 2009 to 38 per $100000 .^{[3]}$ Several studies have shown that the decrease has been most evident in firearm-related homicides ${ }^{[3-5]}$ and that it coincided with the introduction of stricter gun control legislation. One study estimated that more than 4500 lives were saved across five SA cities from 2001 to 2005. ${ }^{[5]}$ Pro-gun interest groups seeking to promote gun ownership and diffusion have attacked these findings, suggesting that stricter gun control was only enacted in 2004 following the publication of regulations pertaining to the Firearms Control Act (FCA) of 2000. They also argue that the 5-year study period ignored a pre-existing downward trend in firearm homicide, and that postmortem figures conflicted with official statistics, such as those presented by Statistics South Africa (SSA).

SA is fortunate to have good coverage of death notification nationally, alongside injury mortality surveillance in certain provinces. In this issue of SAMJ, we draw on SSA's death notifications from 1997 to 2013 to ask the question Where have all the gun deaths gone? ${ }^{(6]}$ We found that in death notification data, the trend in gunshot-related injury deaths - irrespective of intent - was consistent with the findings of other studies. The annual number of firearm deaths was increasing during the 1990s and peaked in 2000, the year in which the FCA was adopted by parliament. Thereafter, the annual number of firearm deaths began to decrease, increasingly following the adoption of the regulations related to the FCA in 2004. The study provides further support for the hypothesis that stricter gun control accounted for the decrease in firearm homicide in SA. The findings are not surprising and echo an expanding body of international evidence. In a recent systematic review, SantaellaTenorio et al. ${ }^{[7]}$ identified 130 studies in 10 countries and found that reductions in firearm deaths were associated with the simultaneous implementation of laws encompassing multiple firearm restrictions in certain countries. The authors cited, alongside examples from the USA, Canada, Australia, New Zealand and Brazil, SA's FCA, which banned certain types of firearm, required training tests for licences, additional licences for each gun owned, increased age requirements for ownership and compulsory background checks.

While mortality data reflect the 'good news' of gun control policy saving lives, they are equally adept at showing the deleterious effects of laxity in its enforcement. A concerning finding from our study was that the decrease in homicide had not been sustained for the duration of the study period. We observed an increasing number of gunshot-related deaths in 2012 and 2013. This is also reflected in the increase in murder reported in recent police crime statistics. The timing of the observed increase in gunshot deaths also corresponds noticeably with the police finalising the fast-tracking of more than a million firearm-related applications between November 2010 and
July 2011, alongside allegations of corruption in the licensing and selling of firearms.

In its series of evidence briefings, the World Health Organization includes reducing access to firearms among its 'best buys' for violence prevention. ${ }^{[8]}$ Although reduced access does not address the root causes of violence, it does, more often than not, reduce the severity and lethality of interpersonal conflict. In a high-violence setting such as SA, there are limited opportunities to effect a significant reduction in violence and homicide at the population level, and to do so relatively quickly. Most evidence-based strategies to reduce violence, such as investing in the early development stages of childhood and increasing positive adult involvement in child monitoring and supervision, establishing positive sociocultural norms, reducing socioeconomic inequalities and improving criminal justice and social welfare systems, all require long-term commitment and investment. As such, they can be difficult to sustain over relatively short political cycles. ${ }^{[9]}$

Reducing firearm mortality by means of stricter gun control is one of the most important short- to medium-term measures to address the burden of violence in SA, while longer-term interventions and policy options take effect. When we have evidence that policies and interventions are working, we need to ensure that they are applied rigorously and consistently. We should be wary of attempts to deny the evidence by groups and individuals promoting their own narrow ideological and commercial interests ahead of the public good.

\section{R Matzopoulos}

South African Medical Research Council Burden of Disease Research Unit, Cape Town, South Africa, and School of Public Health and Family Medicine, Faculty of Health Sciences, University of

Cape Town, South Africa

richard.matzopoulos@mrc.ac.za

1. Prinsloo M, Matzopoulos R, Laubscher R, Myers J, Bradshaw D. Validating homicide rates in the Western Cape Province, South Africa: Findings from the 2009 Injury Mortality Survey. S Afr Med 2016;106(2):193-195. DOI:10.7196/SAMJ.2016.v106i2.10211

2. Bradshaw D, Nannan N, Laubscher R, et al. South African National Burden of Disease Study 2000: Estimates of Provincial Mortality. Cape Town: Medical Research Council, 2004. http://www.mrc.ac za/ bod/estimates.htm (accessed 9 May 2016).

3. Matzopoulos R, Prinsloo M, Pillay-Van Wyk V. Injury-related mortality in South Africa: A retrospective descriptive study of postmortem investigations. Bull World Health Organ 2015;93(5):303-313. DOI:10.2471/BLT.14.145771

4. Abrahams N, Mathews S, Martin LJ, Lombard C, Jewkes R. Intimate partner femicide in South Africa Abrahams N, Mathews S, Martin LJ, Lombard C, Jewkes R. Intimate partner femicide in
in 1999 and 2009. PLoS Med 2013;10(4):e1001412. DOI:10.1371/journal.pmed.1001412

in 1999 and 2009. PLoS Med 2013;10(4):e1001412. DOI:10.1371/journal.pmed.1001412 cities: A retrospective population-based study. Am J Public Health 2014;104(3):455-460. DOI:10.2105 AJPH.2013.310650

6. Matzopoulos RG. Where have all the gun deaths gone? S Afr Med J 2016;106(6):589-591. DOI:10.7196 SAMJ.2016.v106i6.10379

7. Santaella-Tenorio J, Cerdá M, Villaveces A, Galea S. What do we know about the association between firearm legislation and firearm-related injuries? Epidemiol Rev 2016;mxv012. DOI:10.1093/epirev/ mxv012

8. World Health Organization. Violence prevention. The evidence, Geneva: WHO, 2010, http://www. who.int/violence_injury_prevention/violence/4th_milestones_meeting/publications/en/ (accessed 9 May 2016).

9. Matzopoulos R, Bowman B, Mathews S, Myers JE. Applying upstream interventions for interpersonal violence prevention: An uphill struggle in low- to middle-income contexts. Health Policy 2010;97(1):62-70. DOI:10.1016/j.healthpol.2010.03.003

S Afr Med J 2016;106(6):544. DOI:10.7196/SAMJ.2016.v106i6.11034 